6 THE USAGE OF LEAN TECHNOLOGIES' TOOLS AS EFFECTIVE METHOD OF OPTIMIZATION OF PEDIATRICIAN'S WORK IN A SIMULATED CLINIC

Valeria Pavlovna Novikova*, OV Lisovskii, AV Gostimskii, IA Lisitsa, IV Karpatskii, MV Gavschuk, AN Zavyalova, NV Getsko, EO Lisovskaya, MD Prudnikova. St.Petersburg State Pediatric Medical University, Ministry of Healthcare of the Russian Federation, Saint Petersburg, Russia

\subsection{6/archdischild-2021-europaediatrics.6}

Optimization the work of a pediatrician on outpatient appointment, using the principles of lean- technologies in healthcare.

A training center for studying of lean technologies in healthcare has been created at the St. Petersburg State Pediatric Medical University. The purpose of the Center is training doctors and nurses using the lean technology tools, for example, on a simulated clinic in which similar medical processes are simulated like in real clinics. The implementation of the tasks is achieved by conducting business games and factory process in medicine consisting of three stages with the subsequent identification the problems of the primary admission and the optimization the work of specialists. After each round, a debriefing was conducted and medical losses were identified with suggestion for improvement of quality in treatment and examination. Repeated repetition of identical processes in simulated conditions allows to identify losses at all stages of medical care.

The training of doctors and nurses in children's clinics is traditionally carried out at the workplace. Improving the quality and ensuring the availability of medical care to the population determines the necessity to optimize the doctor's work at the initial appointment in the clinic.

The conducting factory process in medicine on the early stages allows to reveal of the lack of a single view about the current and target state of the process, mapping techniques and types of losses and, of course, optimization opportunities. When the factory was re-conducted by similar specialists, the time of admission for children was reduced from 12 to 4 minutes, which allowed to take more time for each patient by optimization the work of doctors and nurses. During the action of factory processes, the waiting time for reception in the hall was also observed. Thus, by redistributing the responsibilities and functions of nurses, it was possible to obtain waiting times in the line, reduced from 45 to 20 minutes for each patient. The learning of lean technologies tools in a simulated clinic helped to understand process optimization skills to use the knowledge in practice.

Using the factory processes and business games in a simulated clinic and the integrated implementation of lean technology tools allowed us to optimize the conditions for the effective and high-quality work of medical staff in clinics and, as a result, to improve the quality of medical care for the patients.

\section{MULTIDISCIPLINARY TREATMENT OF A PATIENT WITH ANOREXIA NERVOSA - CASE REPORT}

Silvana Pleština*, Ivan Begovac, Lena Santric, Veronika Nives Zorić, Irena Bambulović. Department for psychiatry and psychological medicine, School of medicine, University Zagreb, Clinical hospital center Zagreb
Anorexia nervosa is an eating disorder that is fairly common in adolescents. The treatment of this disorder involves a multidisciplinary approach with specialists from a variety of fields including: pediatricians, child and adolescent psychiatrists, psychologists, psychotherapists and nurses. A female adolescent patient with anorexia nervosa- restrictive type, who exhibits an unfavorable course, will be presented in this case report. The patient was treated by pediatricians, as well as child and adolescent psychiatrists and other healthcare professionals, as an inpatient and outpatient numerous times. Unfavorable prognostic factors in this case included: resistance and prolonged duration of symptoms, pronounced psychopathology, lack of motivation for treatment, deficient personality structure, and consequent splitting of good and bad as a defense mechanism. The patient achieved partial stabilization during her last hospitalization on the child and adolescent psychiatry ward. In practice, anorexia nervosa remains a challenge to treat; especially in those cases with an unfavorable course.

\section{DIAGNOSTIC AND SURGICAL TREATMEANT OF NECK CYSTS IN CHILDREN- (POSTER PRESENTATION PREFERRED)}

Duška Markov-Glavaš* , Iva Carević, Nikolina Jurišić. Srebrnjak Children's Hospital

\subsection{6/archdischild-2021-europaediatrics.8}

Midline neck masses are a heterogeneous group of conditions and making the correct diagnosis can sometimes be challenging. The majority of them are caused by congenital abnormalities including: thyroglossal duct cyst, branchial left cyst and dermoid cyst. Thyroglossal duct cyst is the most common, presenting in $2-4 \%$ of all neck masses.

In the abstract 21 cases (52\% boys) of operated congenital cysts are retrospectively analysed between 2016 and 2019 in Children`s Hospital Srebrnjak, Zagreb. Those cysts clinically presented as masses in the midline neck region and they were histologically diagnosed as congenital cysts.

Surgical indications were based on patient history, clinical examination and ultrasound imaginig as well as magnetic resonance.

CASE 1

GIRL, 10 months old

Clinical presentation, preoperative ultrasound and magnet resonance imaging, excision, histology and outcome.

Diagnosis: Dermoid cysts

CASE 2

Boy, 6 years old

Clinical presentation, preoperative ultrasound imaging, excision, histology and outcome.

Diagnosis: Tyroglossal duct cyst

21 cases were reviewed, of which two seemed clinically most challenging and interesting in terms of diagnosis and management have been presented in this abstract.

Congenital abnortmalities are the most common cause of the mass in the midline neck region in children. Although patient history and physical examination is imortant, knowledge of anatomy and embriology is essential for different diagnosis, just like experience in preoperative ultrasound imaging. 